\title{
Image Fusion using Hybrid De-noising and Curve-let Transform
}

\author{
Huma Mir ${ }^{1}$, Tushar Vyas \\ Department of Computer Science and Engineering Vivekananda Global University, Jaipur, Rajasthan, India.
}

\begin{abstract}
In this paper a fuzzy type image-fusion technique using hybridization of Curve let and de-noising based on Pure-let transform is used. The method shows fusion of images and can be used for fusion of multi model image. It is concluded that fusion with advanced single levels offers better fusion quality. This method provides a comparative study between proposed \& literature techniques and validation of the projected algorithm as Peak Signal to Noise Ratio (PSNR), Root Mean Square Error (RMSE).

Keywords: Fuzzy type image, Curve let transform, PSNR, RMSE, Pure-let transform.
\end{abstract}

\section{INTRODUCTION}

In Biomedical Science it is required to expand the data substance of a picture and various enrolled pictures are consolidated together to get image fusion however it is a promising research area. Image fusion gives an effective method to decrease this expanding volume of data by removing all the helpful data from the source pictures [7]. Image fusion makes new pictures that are more usable for the grounds of human/machine insight, and for more remote image processing activities, for example, division, object location or target recognition in fields, for example, remote sensing and medical imaging. Multi sensor image fusion has become a discipline which demands more common formal solutions to a number of relevance cases. Several circumstances in image processing require both high spatial and high spectral data in a solitary picture. This is essential in image processing.

Multi sensor information fusion assumes a basic part in defence in addition to civilian applications as it includes differing qualities of sensors offered and those working in diverse spectral bands. Multi sensor image fusion has turned into a discipline which requests more broad formal answers for various application cases. Image fusion gives an efficacious method for reducing the expanding amount of data while in the meantime removing all the important data from the source pictures. The after effect of the utilization of these methods is an expansion in aggregate sum of information accessible [4, 8]. The main motive of image fusion aside from reducing the data of information is to create new pictures that are more suitable for the motivations behind human/machine recognition and for further image processing errands for example, remote detecting and medical imaging likewise in applications like segmentation, object detection or target recognition. A further broad device in signal and image processing is multi-scale deterioration. In Biomedical Science it is required to build the data substance of an image and various enlisted images are consolidated together to get image fusion however it is ongoing research part.

The important issues in remote sensing and mapping application are fusion of multispectral and panchromatic image [9]. Many image fusion methods and programming tools have been developed. The familiar methods are, such as, the Brovey, the IHS (intensity, Hue, Saturation) colour model, the PCA (Principal Component's analysis) techniques, wavelet method and curve-let technique. Appraisal of the nature of the fused pictures is another essential issue. Wald et al projected an approach with criteria that can be used for assessment the spectral quality of the fused satellite images [7]. If the purpose of image fusion is to create artificial image that are nearer to the reality then, the Brovey, IHS and PCA fusion technique meet this requirement. On the other hand, one drawback of such technique is some distortion of spectral characteristics in the original multispectral images. A solution to this drawback is wavelet transform technique. Image fusion based on wavelet transform technique provides high spectral quality of the fused satellite images. Though, the fused images by wavelet have a huge less spatial data than those by the Brovey, IHS and PCA techniques. In remote sensing applications the spatial information of fused image is an essential feature as much as spectral information of fused image [11]. Specifically, this enhances the efficiency of the picture fusion application, for example, unsupervised image classification. Basically, it is necessary to build up advanced image fusion method so that the fused images have the same spectral resolution as the multispectral images and the same spatial image resolution as the panchromatic image with minimum object.

In this paper, we introduced a new image fusion technique based on the curve-let transformation. The image which is obtained by using curve-let based image fusion technique represents nearly the same feature as the original 
panchromatic image because curve-lets represent edges better than wavelets and the same colour as the original multispectral images because we use the wavelet based image fusion method logically in our algorithm. Apart from this, to provide security to original image and fused image we are using some cryptography techniques. This will secure our image from unauthorized access.

\section{CURVE-LET TRANSFORM}

The basic idea behind curve-let is to signify a curve as a superposition of function of various lengths and breadth obeying the scaling law breadth $\approx$ length $^{2}$. This should be done by first breaking the image into sub-bands, i.e, separating the object into a series of disjoint scales. Each scale is then examined by means of a logical ridgelet transform.

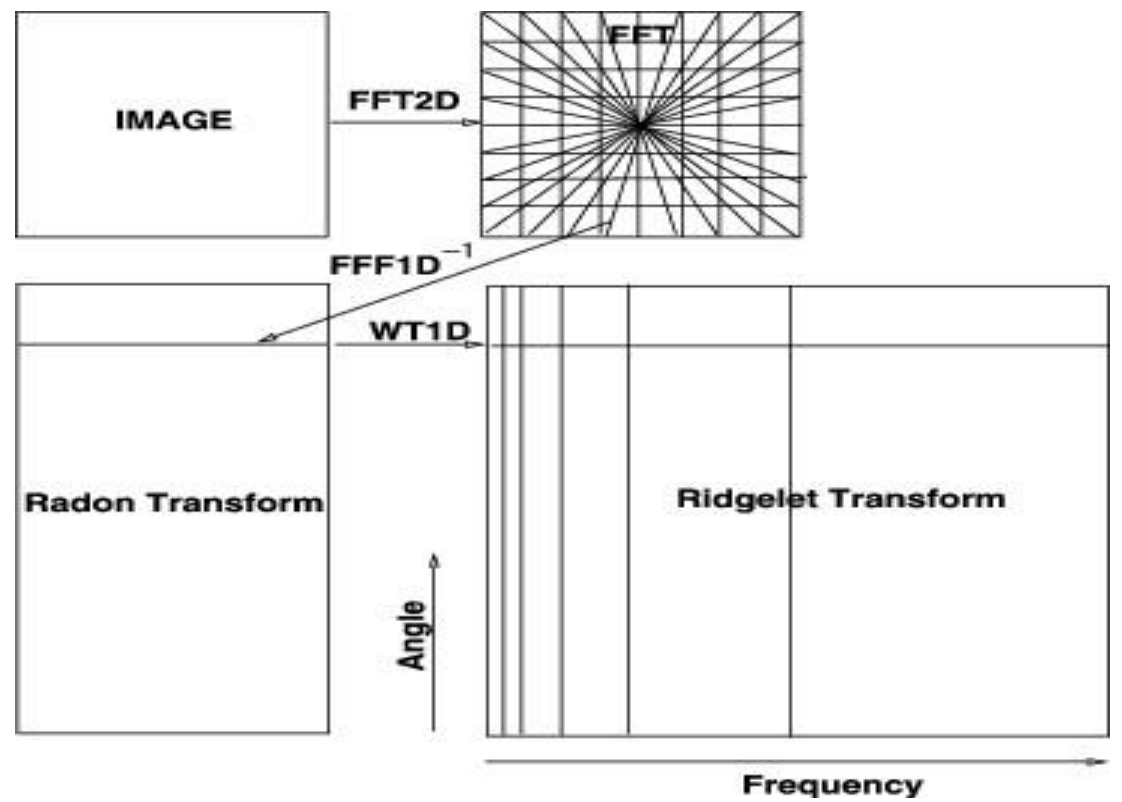

Fig 1: Ridgelet transform flow chart [1]

The 2-D continuous ridgelet transform in $\mathrm{R}^{2}$ can be defined as follows [7, 9]. We choose a smooth univariate function $\psi: \mathrm{R} \rightarrow \mathrm{R}$ with adequate decay and fulfilling the suitable condition.

$$
\int|\psi(\xi)|^{2} /|\xi|^{2} d \xi<\infty
$$

Which holds if, say, $\psi$ has a vanishing mean $\int \psi(t) d t=0$. We will suppose that $\psi$ is normalized so that $\int_{0}^{\infty}|\tilde{\psi}(\xi)|^{2} \xi^{-2} d \xi=1$. For each a $>0$, each $\theta \in[0,2 \pi]$. We also define bivariate ridgelet $\psi_{a, b, \theta}: \mathrm{R}^{2} \rightarrow \mathrm{R}^{2}$ by

$$
\psi_{a, b, \theta^{(x)}}=a^{-1 / 2} \psi\left(\left(x_{1} \cos \theta+x_{2} \sin \theta-b\right) / a\right)
$$

A ridgelet is constant along lines $x_{1} \cos \theta+x_{2} \sin \theta=$ const. Traverse to these ridges it is a wavelet.
Given $\int \in L^{2}\left(R^{2}\right)$, we define its ridgelet coefficients by

$$
\mathfrak{R}_{f}(a, b, \theta)=\int \bar{\psi} a, b, \theta^{(x)} f(x) d x
$$

If, $f \in L^{1}\left(R^{2}\right) \bigcap L^{2}\left(R^{2}\right)$, then we have the exact reconstruction formula.

$$
f(x)=\int_{0}^{2 \pi} \int_{-\infty}^{\infty} \int_{0}^{\infty} \mathfrak{R}_{f}(a, b, \theta) \psi a, b, \theta(x) \frac{d a}{a^{3}} d b \frac{d \theta}{4 \pi}
$$

And a Perceval relation

$$
\int|f(x)|^{2} d x=\int_{0}^{2 \pi} \int_{-\infty}^{\infty} \int_{0}^{\infty}\left|\Re_{f}(a, b, \theta)\right|^{2} \frac{d a}{a^{3}} d b \frac{d \theta}{d \pi}
$$

Hence, like wavelet or Fourier transform, the identity (4) expresses the fact that one can any arbitrary function as a continuous superposition of ridge-let $[2,7]$. Curve-lets are a suitable basis for signifying images (or different functions) which are smooth separated from singularities along smooth curves, where the curves have restricted curvature, i.e. where objects in the picture have a base length 
scale. This property holds for cartoons, geometrical figures, and content. The curve-let change is a multi-scale directional change that permits a practically ideal non versatile sparse representation of items with edges. Curve-let transform was introduced by Candes and Donoho in year 2000 which is suited for objects which is suited for curved surfaces. To analyze local line or curve singularities, a division of the image is considered, and after that ridgelet transform is applied to the obtained sub-images [5]. Curve discontinues are handled efficiently by curve-let transform as they are designed to handle curves using only a small number of coefficients. Curve-let transform was extended to the fields of edge detection and image de-noising.

The algorithm of the curve-let transform of an image $\mathrm{P}$ can be summarized in the following steps:

i. The image $\mathrm{P}$ is split up into three sub hands $\Delta 1, \Delta 2$, and $\mathrm{P} 3$ using the adaptive wavelet transforms.

ii. Tilting is performed on the sub bands $\Delta 1, \Delta 2$.

iii. The discrete Ridgelet transform is performed on each tile of the sub bands $\Delta 1, \Delta 2$

Curve-let transform is a tool for representation of curved shapes in images .The concept of curve-let transform is based on the segmentation of whole image into small overlapping tiles and then applying ridgelet transform on each tile.

\section{Parameters for performance evaluation in image fusion:}

The performance parameters in image fusion algorithms can be evaluated as

i. Root mean square error.

ii. Peak signal to noise ratio.

\section{i. Root Mean Square Error (RMSE):}

Root mean square error is the calculation of the equivalent pixels in the true image and fused image [2]. Error generation will be zero when true and fused images are similar and error generation will increase when dissimilarity between images increase.

$$
R M S E=\sqrt{\frac{1}{M N} \sum_{x=1}^{M} \sum_{y=1}^{N}\left(i_{t}(x, y)-i_{f}(x, y)\right)^{2}}
$$

\section{ii. Peak Signal To Noise Ratio (PSNR):}

PSNR is a measure of increased value when fused and true image are similar and superior value entails superior fusion.

$$
P S N R=20 \log _{10}\left(\frac{l^{2}}{\frac{1}{M N} \sum_{x=1}^{M} \sum_{y=1}^{N}\left(i_{t}(x, y)-i_{f}(x, y)\right)^{2}}\right)
$$

Here ,L indicate the number of gray levels.

\section{Pure-let approach:}

The basic tool is a statistical approximation of the Mean Square Error (MSE) or "risk" among the unknown noiseless image and the processed noisy image. Pure-let has been recently proposed as noise separated scheme from Poisson count images. Specifically, PURE (Poisson unbiased risk estimator) is an impartial estimate, defined in the Haar wavelet domain of the mean squared error among the original image and the estimated image [24].

Pure-let endeavour to approximate the true image from the noisy image by minimizing Poisson unbiased risk estimator (PURE). Pure-let entails inversion of a small size matrix. The main idea of Pure-let based on an optimization of a statistical tool MSE which measures the risk between the noiseless restored image and the noisy image. Minimization of this MSE estimate over a collection of 'acceptable' de-noising process to find the best one, in the sense of the signal to noise ratio (SNR) which is a widespread measure of restoration quality [25].

\section{a. Poisson unbiased risk estimator (PURE):}

In practice we don't have access to the oracle MSE between $\mathrm{x}$ and the estimated $\hat{x}$ but we can use an unbiased estimate of its expected value. Poisson unbiased risk estimate (PURE) which solely depends on the observed image by:

$$
\begin{aligned}
& \text { PURE } \\
& \frac{1}{N}\|F(Y)\|^{2}-\frac{2}{N} \operatorname{dif}_{H} F(Y)+\frac{1}{N}\|x\|^{2}
\end{aligned}
$$

Is an unbiased estimate of the expected MSE i.e.

$$
\begin{aligned}
& \mathrm{E}(\in)=\frac{1}{N} \mathrm{E}\left\{\|F(Y)-X\|^{2}\right\} \\
& \text { Where } \quad \operatorname{dif}_{H} F(Y)=\sum_{n=1}^{N} y_{n} f_{n}\left(y-\alpha e_{n}\right) \text { and } \\
& F(Y)=H^{-T} f(y), e_{n} \text { is the N-dimensional }
\end{aligned}
$$
vector with components $\delta_{K=N}, K=1,2 \ldots \ldots . . . N$.

However, there are two implementation issues. One is caused by the possible ill calculating of the matrix $H^{-1}$. This can be addressed by replacing $H^{-1}$ with the Tikhonov regularized inverse. 


$$
H_{\beta}^{-1}=\left(H^{T} H+\beta P^{T} P\right)^{-1} H^{T}
$$

For some parameters $\beta>0$ and matrix $P \in R^{N} \mathrm{X} R^{N}$.

The other issue arise from the impractical evaluation of the exact PURE. A direct evaluation of dif $\left\{H^{T} F(Y)\right\}$ would require the calculation of $y^{T} H_{\beta}^{-T} F$ for $\mathrm{N}$ perturbed version of the input $y=\left(y-\alpha e_{n}\right)$ for $\mathrm{n}=1,2 \ldots \ldots . . \mathrm{N}$.

Consequently, the PURE unbiased MSE estimate defined in (3.22) is well approximate by:

$$
\operatorname{PURE}_{\text {app }}\{F\}=\frac{1}{N}\|F(y)\|^{2}-\frac{2}{N} y^{T} H_{\beta}^{-T} F(y)+\frac{2 \alpha}{N} y^{T} H \text {. }
$$

\section{b. Pure-let approach:}

In order to find the function $F$ such that $F(y)$ is close to $\mathrm{x}$, we describe the deconvolution process $\mathrm{F}$ as a linear expansion of thresholds (LET).

$$
F(y)=\sum_{k=1}^{k} a_{k} F_{k}(y)
$$

(3.24)

Where $\mathrm{K}<<\mathrm{N}$ is the number of linear coefficients $\mathrm{a}=a=\left[a_{k}\right]_{k \in[1 \ldots \ldots \ldots N]}$ of the LET.

According to the deconvolution problem is reduced to finding the linear coefficients $a_{k}$ by minimizing the PURE defined in equation (3.23).

By substituting (3.23) into (3.24) and performing differentiation over $a_{k}$ this minimization is equivalent to solving the following linear systems of equations.

$$
\sum_{k^{\prime}=}^{k} F_{k}(y)^{T} F_{k^{\prime}}(y) a_{k^{\prime}}=y^{T} H_{\beta}^{-T}\left(F_{k}(y)-\alpha \partial F_{k}(y)\right)
$$

For $k=1,2 \ldots \ldots . . . k$. These equations could be summarized an $\mathrm{Ma}=\mathrm{c}$, where $\mathrm{M}=$ $M=F^{T} F \in R^{k X k}$

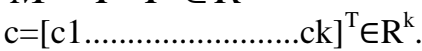

using fuzzy type method as revealed in fig - $3 \& 4$. The fused image is nearly analogous to true image and the error image is approximately zero. Table-1 reveals the fusion quality evaluation metrics. The metrics demonstrated in table with dark font are superior among others. We got almost similar performance by using fusion with 6 level pyramids or some higher values .

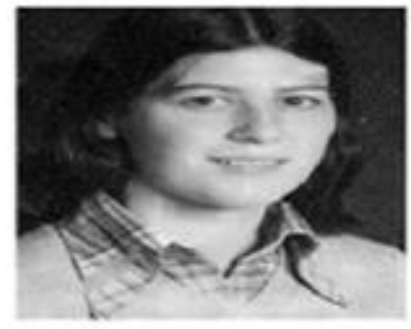

Fig 3: Reference fuzzy type Image

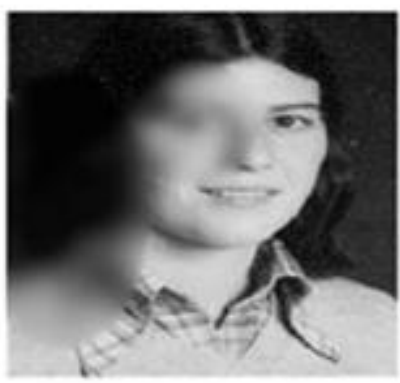

Fig 4: Fuzzy type Image 1

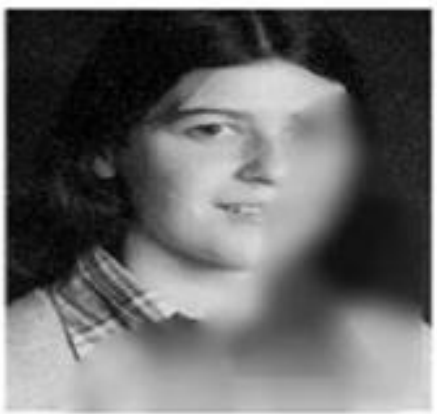

\section{RESULTS AND DISCUSSION}

True Fuzzy type image img1.jpg of size $420 \times 342$ is revealed in fig-2. The 2 images to be fused are produced from the Fuzzy type truth image

Table 1: Proposed Hybrid Technique

\begin{tabular}{|c|l|l|l|l|l|}
\hline \multirow{5}{*}{} & \multicolumn{5}{|c|}{ Pyramid levels } \\
\cline { 2 - 6 } & \multicolumn{1}{|c|}{ Techniques } & 1 & 3 & 5 & 7 \\
\hline \multirow{3}{*}{ RMSE } & DCTPT & 10.0311 & 9.3924 & 9.4921 & 12.7680 \\
\cline { 2 - 6 } & FFTPT & 7.9812 & 7.8937 & 7.9885 & 8.0075 \\
\cline { 2 - 6 } & SPIHT & \multicolumn{5}{|c|}{6.4560} \\
\cline { 2 - 6 } & Curvelet & \multicolumn{5}{|c|}{5.1039} \\
\hline
\end{tabular}




\begin{tabular}{|c|c|c|c|c|c|}
\hline & Curvelet+ PLT & \multicolumn{4}{|c|}{0.0190} \\
\hline \multirow{4}{*}{ PSNR } & DCTPT & 38.1513 & 38.4370 & 38.3912 & 37.1036 \\
\hline & FFTPT & 39.1441 & 39.1920 & 39.1402 & 39.1298 \\
\hline & SPIHT & \multicolumn{4}{|c|}{40.0651} \\
\hline & Curvelet & \multicolumn{4}{|c|}{41.0858} \\
\hline & Curvelet+ PLT & \multicolumn{4}{|c|}{65.3819} \\
\hline
\end{tabular}

\section{CONCLUSION}

An innovative image fusion technique using curve let transform and Pure-let transform has been proposed and its performance is evaluated. We realized better fusion quality by enhancing the fusion levels till 3 . This method can be efficiently used for fusion of images with multi spectral images also. The projected algorithm is easier and efficient to implement for real time applications. This paper also provides a comparative study between proposed $\&$ literature techniques and validation of the projected algorithm as in table 1 .

\section{REFERENCES}

[1]. A. Toet, "A morphological pyramid image decomposition”, Pattern Recogn. Lett. 9(4), 255-261 (1989).

[2]. VPS Naidu and J.R. Raol, "Pixel-Level Image Fusion using Wavelets and Principal Component Analysis - A Comparative Analysis" Defence Science Journal, Vol.58, No.3, pp.338-352, May 2008.

[3]. VPS Naidu, "Discrete Cosine Transformbased Image Fusion", Special Issue on Mobile Intelligent Autonomous System, Defence Science Journal, Vol. 60, No.1, pp.48-54, Jan. 2010.

[4]. Mahendra Kumar et.al., "Digital Image Watermarking using Fractional Fourier transform via image compression", In IEEE International Conference on Computational Intelligence and Computing Research 2013 (IEEE ICCIC-2013), 26-28 Dec., 2013.

[5]. VPS Naidu, "A Novel Image Fusion Technique using DCT based Laplacian Pyramid", International Journal of Inventive Engineering and Sciences (IJIES) ISSN: 2319-9598, Volume-1, Issue-2, January, 2013.

[6]. Rick S. Blum, "Robust image fusion using a statistical signal processing approach", Image Fusion, 6, pp.119-128, 2005.

[7]. Shutao Li, James T. Kwok and Yaonan Wang, "Combination of images with diverse focuses using the spatial frequency", Information fusion, 2(3), pp.167-176, 2001.

[8]. Mahendra Kumar et. Al...'Fuzzy type Image Fusion using SPIHT Image compression technique", International Journal of Engineering Research and Applications (IJERA), (Accepted). (Impact factor: 1.69)
[9]. Mahendra Kumar et. al.," Digital Image Watermarking using Fractional Fourier Transform with Different Attacks" International Journal of Scientific Engineering and Technology, Volume No.3 Issue No.8, Aug. 2014, pp : 1008-1011. ((ISSN : 22771581))

[10]. Mahendra Kumar," Face Recognition using SOM Neural Network with Different Facial Feature Extraction Techniques" International Journal of Computer Applications (IJCA), Vol. 76, No. 3, Aug. 2013, pp.7-11. (Impact factor: 0.821)

[11]. Mahendra Kumar," A Matlab Based High Speed Face Recognition System Using SOM Neural Networks" International Journal of Engineering Research and Applications (IJERA), Vol. 3, Issue 4,July-Aug. 2013, pp.785-790. (Impact factor: 1.69)

[12]. S. Prabha, Dr. M. Sasikala, "Texture Classification Using Curvelet Transform", International Journal of Advancements in Research \& Technology, Volume 2, Issue4, April-2013.

[13]. Rajesh Kumar Kakerda et. Al.,'Fuzzy type Image Fusion using hybrid DCT-FFT based Laplacian Pyramid Transform", 4th IEEE International Conference on Communication and Signal Processing (ICCSP 2015) 02-04 April 2015 - Melmaruvathur, TN, IND.

[14]. M. Kumar et. Al.," Comparative Study Of Different Classifiers Based Speaker Recognition System Using Modified MFCC For Noisy Environment", International Conference Green Computing and Internet of Things (ICGCIoT - 2015) 08-10 Oct., 2015, Delhi, IND.

[15]. Mahendra Kumar et. Al.," Robust Digital Image Watermarking using DCT based Pyramid Transform via image compression", 4th IEEE International Conference on Communication and Signal Processing (ICCSP 2015) 02-04 April 2015 - Melmaruvathur, TN, IND . (Published in IEEExplore)

[16]. Mahendra Kumar et. Al.,'Fuzzy type Image Fusion using hybrid DCT-FFT based Laplacian Pyramid Transform", 4th IEEE International Conference on Communication and Signal Processing (ICCSP 2015) 02-04 April 2015 - Melmaruvathur, TN, IND.. (Published in IEEExplore) 
[17]. Mahendra Kumar et. Al.," Digital Image Watermarking using Hybrid DWT - FFT Technique with Different Attacks", 4th IEEE International Conference on Communication and Signal Processing (ICCSP 2015) 02-04 April 2015 - Melmaruvathur, TN, IND. (Published in IEEExplore)

[18]. Mahendra Kumar et. Al.," Robust Image Fusion based on Optimal Cuve-let Transform", International Conference on Micro-Electronics and Telecommunication Engineering (ICMETE 2016) 22 \& 23, September, 2016, SRM University, Modinagar, Gaziabad, India.

[19]. Mahendra Kumar et. Al.," Image Fusion Based On Hybrid SPIHT and SOMA", International Conference on Micro-Electronics and Telecommunication Engineering (ICMETE 2016) 22 \& 23, September, 2016, SRM University, Modinagar, Gaziabad, India.

[20]. Mahendra Kumar et. al., "Image Fusion based on the Modified Curvelet Transform" In International Conference on Smart Trends for Information Technology and Computer Communications (SmartCom - 2016), Jaipur, India, $6-7$ August, 2016. (Proceeding in CCIS Series of Springer ) (ISSN Number 1865-0929).

[21]. Mahendra Kumar et. Al.," Optimal Image Fusion", International Journal of Computer Applications (IJCA) (Accepted) for October 2016. 